\title{
Why Constructive Relativity Fails
}

\author{
John D. Norton ${ }^{1}$ \\ Department of History and Philosophy of Science \\ Center for Philosophy of Science \\ University of Pittsburgh \\ http://www.pitt.edu/ jdnorton
}

Constructivists, such as Harvey Brown, urge that the geometry of Newtonian and special relativistic spacetimes results from the properties of matter. Whatever this may mean, it commits constructivists to the claim that these spacetime geometries can be inferred from the properties of matter without recourse to spatiotemporal presumptions or with few of them. I argue that the construction project only succeeds if constructivists antecedently presume the essential commitments of a realist conception of spacetime. These commitments can be avoided only by adopting an extreme form of operationalism.

${ }^{1}$ I am grateful for discussion to John Earman, Nick Huggett, Ben Jantzen and Ed Slowik; and to members of the seminar HPS 2534 General Relativity and Gravitation, Fall 2007, Department of History and Philosophy of Science, University of Pittsburgh. Revisions of a first draft benefited greatly from the symposium “Time and Relativity," Institute for Advanced Study, University of Minnesota, October 25-27, 2007, and from discussion with all its participants. They included Katherine Brading, Michel Janssen, Antigone Nounou, Oliver Pooley, Chris Smeenk and especially Harvey Brown, who endured my persistent interrogation with patience and good cheer. However the formulations presented here of Brown's views on space, time and matter are my own. 


\section{Introduction}

In his Physical Relativity: Space-time Structure from a Dynamical Perspective, Harvey Brown (2005) advocates a constructive approach to spacetime theories. The idea is that spacetime theories are essentially matter theories. The familiar spacetime geometries are dependent on properties of matter and induced by them. For example, he urges (pp. vii-viii) that "relativistic phenomena like length contraction and time dilation are in the last analysis the result of structural properties of the quantum theory of matter." Elsewhere ${ }^{2}$ (p.132) he describes the view "defended in this book" as "one is committed to the idea that Lorentz contraction is the result of a structural property of the forces responsible for the microstructure of matter," that

The appropriate structure is Minkowski geometry precisely because the laws of physics of the non-gravitational interactions are Lorentz covariant.

and apparently endorses the idea that "these forces and structures are, indeed, actually responsible for the phenomena, and, hence for space-time having the structure it has."

There is an ambiguity in the use of the terms central to Brown's claim: "result of," "because" and "responsible for." They may be understood in two ways. First, they may carry an explanatory import, so that we are to look to matter theory for an explanation of why spacetime has the structure it has. This is understanding that one finds most easily in the text. There is a strong and frequent emphasis on explanation ${ }^{3}$ and the urging that this explanatory order should not be reversed. (See Section "8.2 Minkowski Spacetime: The Cart or the Horse”.)

There is also a second stronger reading. The terms may carry ontological import. Then spacetime results from matter in the sense that spacetime structures are all really properties of matter; or, more carefully, spacetime supervenes on matter. An important consequence of that supervenience is that there can be no differences in spacetime structure without differences in the

2 Or again (p.8) he reports Bell's attempt at "demonstrating with an appropriate model that a moving rod contracts and a moving clock dilates, because of how it is made up and not because of the nature of its spatio-temporal environment. Bell was surely right." (Brown's emphasis here and in main text.)

3 For example, see also pp. 139-143 for Section 8.3 "What does Absolute Geometry Explain?" and "what is at issue is the arrow of explanation" (p. 142). 
underlying matter. This reading is harder to find, but it is there. Brown asserts (p. 141)“...the space-time structures [of Newtonian theory and special relativity] ... are not real entities in their own right at all" and (p. 142); that the 4-connection of these theories is "a codification of certain key aspects of the behaviour of particles and fields"; and (p. 100) that "I see the absolute geometrical structures of Minkowski space-time as parasitic on the relativistic properties of the dynamical matter fields." These sentiments are captured more vividly in the earlier slogan of Brown and Pooley (2004) of Minkowski spacetime as a "glorious non-entity." This would make Brown's view a form of spacetime relationism, although I will suggest below in Section 6 that it might be more restrictive than familiar forms of relationism.

Brown's readers will decide for themselves how these two claims interact. A natural possibility is that the supervenience claim is the fundamental one; and that the explanatory claims are intended to defend it by denying explanatory power to structures of space, time or spacetime geometries.

Since many details of Brown's text have been addressed elsewhere (for example, Dorato, 2007, Martinez, 2007 and Skow, 2006), my concern in the present note is with just one issue. It is to explore whether the sort of constructivism Brown advocates is a viable alternative to the standard view. Whatever meanings may be intended for the terms mentioned, if the claims are to harbor interesting novelty, they must differ significantly from what I shall label a standard "realist" view of spacetime. In the case of special relativity, that view amounts to:

Realist conception of Minkowski spacetime. ${ }^{4}$

(a) There exists a four-dimensional spacetime that can be coordinatized by the standard coordinates $(\mathrm{x}, \mathrm{y}, \mathrm{z}, \mathrm{t})$

(b) The spatiotemporal interval s between events $(\mathrm{x}, \mathrm{y}, \mathrm{z}, \mathrm{t})$ and $(\mathrm{X}, \mathrm{Y}, \mathrm{Z}, \mathrm{T})$ is a property of the spacetime, independent of the matter it contains, and is given by

$$
\mathrm{s}^{2}=(\mathrm{t}-\mathrm{T})^{2}-(\mathrm{x}-\mathrm{X})^{2}-(\mathrm{y}-\mathrm{Y})^{2}-(\mathrm{z}-\mathrm{Z})^{2}
$$

${ }^{4}$ I use the older formalism since it is enables me to make my points most compactly. A similar development is possible using a more abstract, geometrical approach in which a Minkowski spacetime metric is explicitly introduced. I do not see any gain, however, in this case from its greater complexity. 
When $s^{2}>0$, the interval s corresponds to times elapsed on an ideal clock; when $s^{2}<0$, the interval s corresponds to spatial distances measured by ideal rods (both employed in the standard way).

(c) Material clocks and rods measure these times and distances because the matter

theories that govern them are adapted to the independent geometry of this spacetime.

If constructivism is to be novel, it must contradict this view; it certainly cannot covertly presume all or most of it.

What I will seek to establish below is that if constructivism is to succeed, it must tacitly presume at least a major part of this realist conception. To show this I will recall that this constructivism depends upon a technical result that is assumed tacitly in Brown's book. It is that familiar spacetime geometry can indeed be constructed - that is, inferred-from matter theories. For, if that is not possible, there will be components of spacetime geometry that do not supervene on matter and also cannot be explained by matter theories; that is, they would not, in the language of Brown's text, be a "result of" properties of matter. I will show below that, once one attends to the details of this construction project, it can only succeed if one tacitly assumes much or all of the realist conception.

The construction project will be indicated by sketching its first steps in Sections 2 and 3 below. In Section 4, I will advance my first claim:

The construction project must tacitly assume an already existing spacetime endowed with topological properties, for otherwise it has no way to introduce spatiotemporal coincidences.

In Section 5, I will advance the second claim:

The Lorentz covariance of all matter theories asserts an adaptation between matter and this spacetime akin to the realist's (c), although without (c)'s presumption of the direction of the adaptation of matter to spacetime.

In Section 6, I will advance the third claim:

Constructivists must accept that spatial distances and times elapsed are properties of spacetime as asserted in (b), on pain failing to reconstruct traditional spacetime geometry and also having to accept an extreme form of operationism in which quantities have values only if they are actually measured.

The claims made and the discussion throughout will be limited to Newtonian theory and special relativity. For once one makes the transition to general relativity, the distinction between matter 
and spacetime becomes harder to draw; the spacetime metric now represents both the geometric properties of spacetime as well as the gravitational field.

Finally, the discussion below will eschew the explanatory issues that have dominated discussion elsewhere. For they seem only to lead to futile disputes over just what it means to explain, over which is the better explanation and, most opaquely, which is the "real" explanation. While a full understanding of constructivism may eventually require clarification of these explanatory concepts, my goal here is to show that we can advance our analysis of constructivism by paying attention to its non-explanatory aspects. ${ }^{5}$

\section{The Construction Project}

The strongest form of constructivism presumes the success of the following project, which has been specialized to special relativity for concreteness:

Construction of Minkowski spacetime. It is possible to recover the geometry of Minkowski spacetime from Lorentz covariant matter theories devoid of spatiotemporal presumptions.

The essential condition in this specification is that the matter theories must be devoid of spatiotemporal assumptions. Without it we would, to one degree or another, simply be returning

5 To see how rapidly these concerns lead to stalemates, consider how we might explain the puzzle posed in Brown (p. 24) that free particles in relativity theory follow spacetime geodesics. If we demand a causal explanation that draws on a primitive notion of causation, then we are conjuring up a dubious level of causal metaphysics that must be supposed to be antecedent to science. Successful explication of that notion of cause has eluded us for millennia (Norton, 2003). Or we may understand the use of causal language as merely a gloss on the use of a deeper theory. In conformity with this latter view, Brown (pp. 161-63) offers as an explanation the deduction of the geodesic principle from the law of conservation of matter in general relativity. That renders the view of explanation deductive-nomological. Then our judgments of which are the better explanation may dissolve into disputes over which explanans has fewer assumptions. To balance Minkowski geometry's one assumption, is the competing "brute fact" (p. 143) of the Lorentz covariance of all matter theories one assumption or many, one for each matter theory? 
spatiotemporal notions presumed at the start. The more that a version of constructivism presumes spacetime notions, the less it differs from the realist view.

For this strong form, it is easy to envisage what a stripped down matter theory, devoid of spatiotemporal notions, would look like. Take any Lorentz covariant matter theory expressed in the standard coordinates (x, y, z, t) of (1), such as Maxwell's electrodynamics, Dirac's electon theory or the quantum field theory of selected interactions. Discard all spatiotemporal presumptions and interpretations for these coordinates. They are now merely parameters whose meaning is given entirely by their role in the particular matter theory in which they appear. Since we have many matter theories that I will conventionally number $1,2,3, \ldots$, we should indicate to which matter theory a set of four parameters belongs by using subscripts:

$$
\left(\mathrm{x}_{1}, \mathrm{y}_{1}, \mathrm{z}_{1}, \mathrm{t}_{1}\right),\left(\mathrm{x}_{2}, \mathrm{y}_{2}, \mathrm{z}_{2}, \mathrm{t}_{2}\right),\left(\mathrm{x}_{3}, \mathrm{y}_{3}, \mathrm{z}_{3}, \mathrm{t}_{3}\right), \ldots
$$

The presumption that will be key to the reconstruction is the Lorentz covariance of all matter theories. It is a "brute fact," according to Brown (p. 143). ${ }^{6}$ It asserts that the equations of each matter theory remain unchanged under a Lorentz transformation of the parameters. This group includes

$$
\mathrm{t}_{\mathrm{i}}{ }^{\prime}=\gamma\left(\mathrm{t}_{\mathrm{i}}-\mathrm{vx}_{\mathrm{i}}\right) \quad \mathrm{x}_{\mathrm{i}}{ }^{\prime}=\gamma\left(\mathrm{x}_{\mathrm{i}}-\mathrm{vt}_{\mathrm{i}}\right) \quad \mathrm{y}_{\mathrm{i}}{ }^{\prime}=\mathrm{y}_{\mathrm{i}} \quad \mathrm{z}_{\mathrm{i}}{ }^{\prime}=\mathrm{z}_{\mathrm{i}}
$$

where $\gamma=\left(1-\mathrm{v}^{2}\right)^{-1 / 2}$ and $\mathrm{v}$ is an arbitrary parameter such that $-1<\mathrm{v}<1$; spatiotemporal translations

$$
\mathrm{t}_{\mathrm{i}}{ }^{\prime}=\mathrm{t}_{\mathrm{i}}-\mathrm{T} \quad \mathrm{x}_{\mathrm{i}}{ }^{\prime}=\mathrm{x}_{\mathrm{i}}-\mathrm{X} \quad \mathrm{y}_{\mathrm{i}}{ }^{\prime}=\mathrm{y}_{\mathrm{i}}-\mathrm{Y} \quad \mathrm{z}_{\mathrm{i}}{ }^{\prime}=\mathrm{z}_{\mathrm{i}}-\mathrm{Z}
$$

where T, X, Y, Z are arbitrary constants; and spatial rotations. There is a corresponding transformation for the other quantities, such as field intensities, of the matter theory. For notational convenience, we denote these quantities communally as " $\mathrm{Q}_{\mathrm{i}}\left(\mathrm{x}_{\mathrm{i}}, \mathrm{y}_{\mathrm{i}}, \mathrm{z}_{\mathrm{i}}, \mathrm{t}_{\mathrm{i}}\right.$ )" for the $\mathrm{i}$-th matter theory. Their transformation rule is

$$
\mathrm{Q}_{\mathrm{i}}{ }^{\prime}\left(\mathrm{x}_{\mathrm{i}}{ }^{\prime}, \mathrm{y}_{\mathrm{i}}{ }^{\prime}, \mathrm{z}_{\mathrm{i}}{ }^{\prime}, \mathrm{t}_{\mathrm{i}}{ }^{\prime}\right)=\mathrm{L}(\mathrm{v}) \mathrm{Q}_{\mathrm{i}}\left(\mathrm{x}_{\mathrm{i}}, \mathrm{y}_{\mathrm{i}}, \mathrm{z}_{\mathrm{i}}, \mathrm{t}_{\mathrm{i}}\right)
$$

for $\mathrm{L}(\mathrm{v})$ a suitable operator.

This is the familiar "passive" formulation of Lorentz covariance. It is equivalent to the "active" form, which will be more useful below. To arrive at it, presume that we have some

6 "In the dynamical approach to length contraction and time dilation..., the Lorentz covariance of all the fundamental laws of physics is an unexplained brute fact." 
configuration of structures $\mathrm{Q}_{\mathrm{i}}\left(\mathrm{x}_{\mathrm{i}}, \mathrm{y}_{\mathrm{i}}, \mathrm{z}_{\mathrm{i}}, \mathrm{t}_{\mathrm{i}}\right)$ admitted by the $\mathrm{i}$-th matter theory. It follows that $\mathrm{Q}_{\mathrm{i}}{ }^{\prime}\left(\mathrm{x}_{\mathrm{i}}{ }^{\prime}, \mathrm{y}_{\mathrm{i}}{ }^{\prime}, \mathrm{z}_{\mathrm{i}}{ }^{\prime}, \mathrm{t}_{\mathrm{i}}{ }^{\prime}\right)$, where transformed parameters are used, is also admitted by the $\mathrm{i}$-th matter theory. All that is needed for this admission is the appropriate functional dependence of the quantities $\mathrm{Q}_{\mathrm{i}}{ }^{\prime}$ on the parameters $\mathrm{x}_{\mathrm{i}}{ }^{\prime}, \mathrm{y}_{\mathrm{i}}{ }^{\prime}, \mathrm{z}_{\mathrm{i}}{ }^{\prime}, \mathrm{t}_{\mathrm{i}}{ }^{\prime}$. So we learn that the same structures $\mathrm{Q}_{\mathrm{i}}{ }^{\prime}\left(\mathrm{x}_{\mathrm{i}}, \mathrm{y}_{\mathrm{i}}, \mathrm{z}_{\mathrm{i}}\right.$, $\left.t_{i}\right)$, now considered as functions of the original parameters $x_{i}, y_{i}, z_{i}, t_{i}$, are also admitted by the $i-$ th matter theory. This $\mathrm{Q}_{\mathrm{i}}{ }^{\prime}$ is the Lorentz boost of the original $\mathrm{Q}_{\mathrm{i}}$ and both are related to the same parameter set $\left(\mathrm{x}_{\mathrm{i}}, \mathrm{y}_{\mathrm{i}}, \mathrm{z}_{\mathrm{i}}, \mathrm{t}_{\mathrm{i}}\right)$. Informally, the new structure is just the old one "boosted into motion at speed $\mathrm{v}$ in the $\mathrm{x}$ direction," but that interpretation is not yet available since we have not yet constructed spatiotemporal quantities.

\section{Clocks}

How is the construction process to proceed? The simplest way is to identify structures within a matter theory that perform as clocks and rods and show that they induce the spacetime interval (1). That simple approach will not always be possible. Some matter theories do not straightforwardly admit clock-like or rod-like structures. An example is Maxwell's electrodynamics, for none of its localized structures are stable. It must be coupled with another theory to produce such structures. We might also wonder how the structures might arise in quantum field theory.

For expediency, let us just assume the simple case, that our matter theory does admit stable clocks and rods. In that case, a clock "at rest" (speaking informally only, since the spatiotemporal notions of rest and motion have not yet been defined) in matter theory 1 is this. It is a localized structure "Clock $\left(\mathrm{x}_{1}, \mathrm{y}_{1}, \mathrm{z}_{1}, \mathrm{t}_{1}\right)$," in the sense that it is associated with a small range of parameter values $\left(\mathrm{x}_{1}, \mathrm{y}_{1}, \mathrm{z}_{1}\right)$, and its states cycle with a period $\tau$ as a function of $\mathrm{t}$. That is

$$
\operatorname{Clock}\left(\mathrm{x}_{1}, \mathrm{y}_{1}, \mathrm{z}_{1}, \mathrm{t}_{1}+\tau\right)=\operatorname{Clock}\left(\mathrm{x}_{1}, \mathrm{y}_{1}, \mathrm{z}_{1}, \mathrm{t}_{1}\right)
$$

for all $\mathrm{t}_{1}$. Consider the straight ${ }^{7}$ segment of a spacetime curve connecting points $\left(\mathrm{x}_{1}, \mathrm{y}_{1}, \mathrm{z}_{1}, \mathrm{t}_{1}\right)$ and $\left(\mathrm{x}_{1}, \mathrm{y}_{1}, \mathrm{z}_{1}, \mathrm{t}_{1}+\mathrm{T}\right)$, for any $\mathrm{T}$. This clock will assign a time $\mathrm{T} / \tau$ interval to it. Without loss of generality, we can rescale these time assignments to agree with coordinate differences simply by

7 That is, the curve whose image points are $\left\{\left(\mathrm{x}_{1}, \mathrm{y}_{1}, \mathrm{z}_{1}, \alpha \mathrm{t}_{1}\right)+\left(\mathrm{x}_{1}, \mathrm{y}_{1}, \mathrm{z}_{1},(1-\alpha)\left(\mathrm{t}_{1}+\mathrm{T}\right)\right): 0 \leq \alpha \leq 1\right\}$. 
multiplying by $\tau$. This merely amounts to the selection of a conventional unit of measure. So we infer

Temporal interval from $\left(\mathrm{x}_{1}, \mathrm{y}_{1}, \mathrm{z}_{1}, \mathrm{t}_{1}\right)$ to $\left(\mathrm{x}_{1}, \mathrm{y}_{1}, \mathrm{z}_{1}, \mathrm{t}_{1}+\mathrm{T}\right)=\mathrm{T}$

From the active form of Lorentz covariance, it now follows 8 that the same time interval must be assigned to the Lorentz boost of this segment. So we have

$$
\begin{aligned}
& \text { Temporal interval from }\left(\gamma\left(\mathrm{x}_{1}-\mathrm{vt}_{1}\right), \mathrm{y}_{1}, \mathrm{z}_{1}, \gamma\left(\mathrm{x}_{1}-\mathrm{vt}_{1}\right)\right) \text { to } \\
& \left(\gamma\left(\mathrm{x}_{1}-\mathrm{v}\left(\mathrm{t}_{1}+\mathrm{T}\right)\right), \mathrm{y}_{1}, \mathrm{z}_{1}, \gamma\left(\mathrm{x}_{1}-\mathrm{v}\left(\mathrm{t}_{1}+\mathrm{T}\right)\right)\right)=\mathrm{T}
\end{aligned}
$$

A short piece of algebra affirms that these two assignments of temporal interval conform to the expression (1) for the spacetime interval. Suitable combinations of Lorentz boosts, translations and rotations enable recovery of the full content of the expression (1) for the spacetime interval for the case of $s^{2}>0$. Informally speaking, that case is the metrical structure of the future and past light cone.

This construction is quite robust. We could have used clocks from other matter theoriesmatter theories $2,3, \ldots$ - and returned the same result as long as we calibrate them to use the same units. Analogously we could construct physical rods and recover the remainder of the expression (1) for the spatiotemporal interval.

\section{The Spacetime Presumed}

Congenial as the sketch of the last section has been, we have progressed much too fast. Buried within it, in one form or another, is most if not all of the realist's conception of Minkowski spacetime. To begin, the construction presumes that the parameters of each matter theory $\left(\mathrm{x}_{1}, \mathrm{y}_{1}, \mathrm{z}_{1}, \mathrm{t}_{1}\right),\left(\mathrm{x}_{2}, \mathrm{y}_{2}, \mathrm{z}_{2}, \mathrm{t}_{2}\right),\left(\mathrm{x}_{3}, \mathrm{y}_{3}, \mathrm{z}_{3}, \mathrm{t}_{3}\right), \ldots$ refer to the same events of spacetime. For example, we presume that clocks from different matter theories will return the same expression (1) for the spacetime interval. That presumes that the origin of the parameters $\left(x_{1}, y_{1}, z_{1}, t_{1}\right)=(0$,

\footnotetext{
8 Some details for this inference: The Lorentz transformation (2c) assures us that the clock system will execute the same number of periods in the original and boosted segment. We presume that times assigned to a segment are scalars. As a result, the quantity dT/d $\alpha$, where $\alpha$ is defined in the previous note, is also a scalar and is the quantity $\mathrm{Q}_{\mathrm{i}}$ of the earlier definition of active Lorentz covariance.
} 
$0,0,0)$ of matter theory 1 refers to the same event in spacetime as the origin of the parameters $\left(\mathrm{x}_{2}, \mathrm{y}_{2}, \mathrm{z}_{2}, \mathrm{t}_{2}\right)=(0,0,0,0)$ of matter theory 2 . So, for example, we could have a clock from matter theory 1 measure the temporal interval from the event $(0,0,0,0)$ to $(\mathrm{x}, 0,0, \mathrm{t})$ and clock from matter theory 2 measure the temporal interval along the segment from the same event $(0,0$, $0,0)$ to $(-x, 0,0, t)$.

This presumption of sameness of referent for the two sets of parameters amounts to a notion of spacetime coincidence of the associated systems. Nothing in the specification of the construction project of Section 2 licensed that notion of spacetime coincidence. For to presume spacetime coincidences is to presume spatiotemporal notions that were explicitly disavowed.

To see that the presumption of spacetime coincidences is contingent and cannot be presumed, consider the case of the internal spin spaces of two spin half particles. Each particle will have parameters associated with it that pick out a point in its own internal spin space. The equality of the parameters, does not amount to a coincidence of the particles. The equality just indicates an equality of their spin angular momenta.

One might imagine two responses. First, one might wonder whether spacetime coincidences are recoverable for two distinct matter fields through their interactions. Imagine that their combined Hamiltonian includes a distance based coupling term. Might that justify the notion that each of their parameters designate the same events in spacetime? For they interact more intensely the more closely they approach in spacetime. The same example of internal spin spaces shows us that this inference does not succeed. It is standard to write spin-spin coupling terms in Hamiltonians, where the coupling energy depends on the closeness of the spin parameters, without thereby assuming that sameness of the parameters betokens spatiotemporal coincidence. In any case, interactions are of no help if we consider two fields that do not interact; we cannot, on these grounds, preclude the possibility that each inhabits its own spacetime.

Second, one might wonder if we should not just formulate all actual matter theories (including those presently unknown to us) from the start with just one set of parameters (x, y, z, t). Then, every time these parameters appear in the context of one or other type of matter, they are always the same parameters. Therefore, they must have the same referent. This escape fails as well, for it simply presumes that the properties of matter are such that all of its forms can employ the very same parameter set $(x, y, z, t)$. As a contingent fact, that is correct if these parameters are spacetime coordinates. However a contingent fact like this cannot presumed in 
advance if one is disavowing all spatiotemporal notions and seeking to infer them. In the case of the internal spin space, one could not use just one parameter set for the two distinct spin spaces of two particles, or at least not without some device that distinguishes the internal space to which each belongs.

Hence the strongest form of the construction project sketched in Section 2 above fails. One cannot recover the unique four-dimensional spacetime (a) of the realist's conception of spacetime except by presuming it at the start. In effect, all the constructions of Section 3 presumed a single spacetime with topological properties and coordinates $(x, y, z, t)$. This does not augur well for constructivists who seriously believe that Minkowski spacetime is a "glorious non-entity."

\section{Lorentz Covariance is a Property of a Matter Theory and Spacetime}

The all-important "brute fact" of Lorentz covariance of all matter theories is not simply the expression of a property of matter. Rather it is the expression of a property of matter and spacetime taken together, a statement of a relation between them. For, in the mode of presentation adopted here, Lorentz covariance is a restriction on how matter may depend functionally on the parameters $(\mathrm{x}, \mathrm{y}, \mathrm{z}, \mathrm{t})$, where these parameters have now been revealed to be particular sorts of coordinates of a spacetime.

To say it differently, the Lorentz covariance of all matter theories expresses a property of mutual adaptation of the matter and the spacetime. This condition is close to the condition (c) of the realist's conception of Minkowski spacetime in Section 1. Where (c) is stronger in that it specifies a direction of adaptation: in its metrical properties, matter is adapted to spacetime. We shall see in the next section that this directed adaptation is difficult for constructivists to avoid.

\section{Spatial Distances and Times Elapsed are Properties of Spacetime}

The Lorentz covariance of matter theories is really a property of matter and spacetime taken together. What of the spatial distances and time elapsed that are recovered from the requirement of Lorentz covariance? Are they properties of spacetime, of matter, or of both? 
Very familiar results strongly suggest - but do not prove - that the spatial distances and times elapsed are properties of the spacetime and that the matter of a Lorentz covariant matter theory is merely an instrument used to measure them. The result is that the construction of the metrical structure of a Minkowski spacetime (1) by means of Lorentz covariance, such as in Section 3 above, depends upon no particular feature of the matter theories governing the clocks and rods. We needed nothing from the matter theory beyond its Lorentz covariance and that the theory can assign a scalar magnitude to just one segment of spacetime. It then follows that that the only admissible metrical structure is (1).

This is evident already in the construction of Section 3. For, let a matter theory assign by any means at all, unit time elapsed to the segment joining $(0,0,0,0)$ and $(0,0,0,1)$. If that matter theory is Lorentz covariant, then assignments of times must be preserved under Lorentz boosts as well as spatiotemporal translations and spatial rotations. These transformations can be used to populate the spacetime and, as before, we recover the expression for the spatiotemporal interval (1), at least for the future and past light cones. ${ }^{9}$

These results are familiar since they reproduce a line of argument extending back to Einstein. A recent, persuasive formulation of the argument form is found in Janssen's notion of a common origin inference or "COI" (Janssen, 2003; Balashov and Janssen, 2003). Reasoning with COIs is commonplace. When we see many identical footprints on the sandy beach, we immediately infer to the existence of the foot that produced them, their common origin. We do not declare the presence of the many footprints a brute fact beyond which we cannot infer. When we find that every planet orbits the sun under the influence of an inverse square law, we infer to their common origin, a potential field that fills the space around the sun, even those parts where there are no planets. Similarly when all matter theories return spatio-temporal measurements all

9 To complete the populating of the spacetime, one needs one further property, the summation property that the time elapsed along a segment $\mathrm{AC}$ is equal to the sum of the times elapsed along segments $\mathrm{AB}$ and $\mathrm{BC}$, where $\mathrm{B}$ is an event on the segment between $\mathrm{A}$ and $\mathrm{C}$. Divide the segment $(0,0,0,0)$ to $(0,0,0,1)$, say, to $\mathrm{n}$ parts $(0,0,0,0$,$) to (0,0,0,1 / \mathrm{n}) ;(0,0,0,1 / \mathrm{n})$ to $(0,0,0,2 / \mathrm{n})$; ... . It now follows by application of time translation (2b) that all $\mathrm{n}$ parts have the same time elapsed interval; from summation that they sum to 1 ; and therefore that the time elapsed in each is $1 / \mathrm{n}$. 
of which conform to a Minkowski spacetime geometry, that is strong evidence that they have a single, common origin, the Minkowski spacetime that they are measuring.

Thus, when one sees the near complete independence of the spatial distances and times elapsed from the specifics of the matter theories, it is hard to resist the realist's assertion (b) that these spaces and times are properties of the spacetime and that (c) matter is merely providing a means of measuring these properties.

A determined constructivist can resist, but only at some cost. That cost comes through the need to renounce a major part of the goal of the construction project and, worse, to commit to an extreme form of operationism in which quantities are declared to have no values unless they are actually measured.

To see this, imagine some part of spacetime that is either devoid of matter or hosts a static matter distribution. In this part of spacetime, we can select two non-coincident timelike separated events A and B such that nothing changes as we pass along the straight segment of spacetime connecting them. In the ordinary realist's conception, we would say that some time elapses between them. What can a constructivist say? There are no material clocks actually present measuring the time elapsed. For there is either no matter present or no change in the matter present as we pass from A to B. So the constructivist has no material basis for the recovery of a time change. If times elapsed are to supervene on matter, or more vaguely to be a result of the properties of matter, then the absence of any change in the matter entails that there is no change in times elapsed. 10

At best the constructivist can say that, counterfactually, had a clock been transported to this part of spacetime and allowed to pass between the events, then it would have read the appropriate time elapsed. If the constructivist's account of time elapsed depends upon these counterfactual scenarios, then of what is the time elapsed a property? Properties, such as elapsed

10 What of the t parameter introduced in Section 2 above? Is it the time elapsed in the usual sense of Minkowski spacetime geometry for this static case? It is not. Since the t parameter was introduced with no antecedent temporal meaning, we must allow the possibility that changes in it correspond to no physical changes. If times elapsed are to be a result of the properties of matter, then the fact that the properties of the matter are the same for all values of $t$ entails that there is no physical change associated with changes of $\mathrm{t}$. 
times, must be carried by something. The fictional clock envisaged is no real thing, but merely something that could have been.

The time elapsed is a property of spatiotemporal segment connecting the events A and B, just as the realist asserts in (b). It is the only thing there to bear the property. ${ }^{11}$

It seems to me that a constructivist has one escape. That is to deny that any time does elapse between events A and B. The constructivist must, in effect, say that the entire scenario envisaged is impossible. The notion that there can be a part of spacetime without matter or a part of spacetime with static matter is simply a confusion on the part of realists. It makes no sense to talk of time in such scenarios. ${ }^{12}$

To adopt this escape commits the constructivists to renouncing a significant part of spacetime theory. In ordinary physical theorizing, it is not uncommon to talk of parts of Minkowski spacetimes empty of matter. Correspondingly, a familiar, elementary case is a Minkowski spacetime harboring a single electric charge and its associated electrostatic field. Both of these must be dismissed as confusions. Many more such standard examples must be dismissed as confusions. Imagine a Minkowski spacetime with two non-interacting, identical clocks in it, at relative rest. It is standard to assume that they tick at the same rate, even though no material processes implement that fact. It could be expressed materially if we found that light signals emitted from the first clock at times $1,2,3, \ldots$ arrived at the second at times 100, 101, $102, \ldots$; and conversely. But there are no such light signals to carry the property of agreement of

11 If one presumes that spatial distances must be measured by rods that have some spatial inhomogeneity corresponding, say, to the markings on a ruler or the joins between successively laid out rulers, then an analogous problem can be described for spatial distances.

12 Brown (p. 101) seems to assert that there can be no time without material change. He writes that "Time, at its most fundamental level, has something to do with change, and change is not an obvious feature of the Malament world [which has an inertial worldline and Minkowski causal structure only]." Also: “The conformal light-cone structure is in itself timeless. It has no nontrivial dynamics." 
rates and, apparently, no way to assign constructive meaning to it. ${ }^{13}$ The realist, however, understands their ticking at the same rate merely to amount to their measuring the common time they inhabit.

The outcome is that the constructivist is not reconstructing our familiar spacetime geometry; the constructivist is extensively correcting it. The corrections are stronger than a spacetime relationist might require. For the latter, time can still exist in a spacetime filled with a static matter distribution. Time exists as relation between the matter of identical time slices; it does not have to be induced by material changes between the timeslices. What justification can the constructivist give for the corrections? The assertion must be that it makes no sense to speak of times elapsed unless a clock or the change in some material process actually measures the times elapsed. This is the extreme form of operationism mentioned.

If constructivists do not adopt this extreme view, then times elapsed and spatial distances must be recognized as properties of spacetime. It now follows that the adaptation between matter and spacetime discussed in Section 5 is an adaptation of matter to spacetime, as (c) of the realist's conception asserts.

\section{Conclusion}

If constructivists are to succeed in their project of fully reconstructing familiar spacetime geometries from matter theories, then they must presume the basic views of the realist conception of spacetime, which amounts to a complete failure of the project. They can only avoid this failure by offering to reconstruct just a part of the familiar spacetime geometry and dismissing the remaining part as an illicit confusion through an extreme form of operationism.

The analysis above took as its target a thorough-going form of constructivism. One can certainly conceive more modest versions that presume some spatiotemporal notions and not others, although the resulting constructivist view becomes less interesting the more spatiotemporal properties it assumes. A natural, partial view might accept the topological space (a) of the realist's conception, but none of the metrical properties of (b) and (c). Nonetheless, the

13 In conformity with this last conclusion, Brown (p. 20) finds that there is no privileged notion of simultaneity between free particles in Newtonian theory. "It follows that Newtonian simultaneity is a by-product of the introduction of forces into the theory." 
same pressures described will drive that more modest constructivist to the realist's (b) and (c) as well.

In addition, advocates of any such partial view must establish that the selection of spatiotemporal presumptions is consistently done. For example, one could probably not posit that rest lengths of rods are properties of the spacetime, but that contractions of moving rods are properties of the matter of the rods. For positing the former may well commit the theorist to the latter as a property of the spacetime. The burden of showing consistency lies with the constructivist.

\section{References}

Balashov, Yuri and Janssen, Michel (2003) "Presentism and Relativity," British Journal for the Philosophy of Science 54, 327-346.

Brown, Harvey R. (2005) Physical Relativity: Space-time Structure from a Dynamical Perspective. Oxford: Clarendon.

Brown, Harvey R and Pooley, Oliver (2004) "Minkowski space-time: a glorious non-entity," http://philsci-archive.pitt.edu/archive/00001661/

Dorato, Mauro (2007), "Relativity Theory between Structural and Dynamical Explanations," International Studies in the Philosophy of Science, 21, pp. 95-102.

Janssen, Michel (2003) “COI Stories: Explanation and Evidence in the History of Science," Perspectives on Science, 10, pp. 457-522.

Norton, John D. (2003)"Causation as Folk Science," Philosophers' Imprint Vol. 3, No. 4 http://www.philosophersimprint.org/003004/; reprinted as Ch. 2, H. Price and R. Corry, Causation and the Constitution of Reality. Oxford University Press, 2007

Martinez, Alberto (2007), "There's no pain in the Fitzgerald contraction is there?" Studies in History and Philosophy of Modern Physics, 38, pp. 209-15.

Skow, Bradford (2006), Review of Brown, Physical Relativity. Notre Dame Philosophical Reviews. 2006.05.11 http://ndpr.nd.edu/review.cfm?id=6603 